\title{
Numerical Study of Random Periodic Lipschitz Shadowing of Stochastic Differential Equations
}

\author{
Qingyi Zhan $(\mathbb{1})^{1}$ and Xiangdong Xie $\mathbb{( i D}^{2}$ \\ ${ }^{1}$ College of Computer and Information Science, Fujian Agriculture and Forestry University, Fuzhou 350002, China \\ ${ }^{2}$ Ningde Normal University, Ningde, Fujian 352100, China \\ Correspondence should be addressed to Qingyi Zhan; zhanqy@lsec.cc.ac.cn
}

Received 10 July 2017; Accepted 10 October 2017; Published 11 January 2018

Academic Editor: Josef Diblik

Copyright (c) 2018 Qingyi Zhan and Xiangdong Xie. This is an open access article distributed under the Creative Commons Attribution License, which permits unrestricted use, distribution, and reproduction in any medium, provided the original work is properly cited.

\begin{abstract}
This paper is devoted to a new numerical approach for the possibility of $(\omega, L \delta)$-periodic Lipschitz shadowing of a class of stochastic differential equations. The existence of $(\omega, L \delta)$-periodic Lipschitz shadowing orbits and expression of shadowing distance are established. The numerical implementation approaches to the shadowing distance by the random Romberg algorithm are presented, and the convergence of this method is also proved to be mean-square. This ensures the feasibility of the numerical method. The practical use of these theorems and the associated algorithms is demonstrated in the numerical computations of the $(\omega, L \delta)$-periodic Lipschitz shadowing orbits of the stochastic logistic equation.
\end{abstract}

\section{Introduction}

The investigation of random periodic orbits at large and in specific stochastic differential equations (SDEs) is a difficult dynamical problem [1]. In general, numerical computation is still one of the most feasible methods of studying random periodic orbits of chaotic systems of SDEs whose applications describe many natural phenomena in meteorology, biology, and so on $[2,3]$.

Due to the sensitivity of the initial value and random noise pumped into the systems constantly, it is difficult to expect that a particular solution of chaotic systems of SDEs can be well approximated by a numerical solution for any given length of time. Therefore, it is always difficult to infer rigorously the existence of a random periodic orbit from the numerical computations. Shadowing property has an important position in theory and application of random dynamical systems (RDS), especially in the numerical simulations of chaotic systems of SDEs. We present here a new method for establishing the existence of a true random periodic orbit of SDEs which lies near a computed random periodic orbit.

In this work the main motivations are twofold. On the one hand, it follows from the classical results about random periodic solutions of SDEs $[1,4]$ that the numerical simulation of random periodic solution has been performed by the author; see [5] and references therein. Here random periodic solution is a special class of periodic solution with random noise inputted, and the detail is shown as Remark 1. This provides the foundation of numerical analysis. On the other hand, it has been inspired by our earlier work $[6,7]$ on shadowing orbits of SDEs where we establish random shadowing in a rather general setting.

For example, the conditions which can assure the stochastic shadowing in a class of SDEs have been constructed in $[6,7]$. Liu et al. have made useful contributions to the numerical analysis of RDS [4, 8, 9]. There are some work on shadowing orbits of discrete random dynamical systems generated by random iterations $[10,11]$. As we know, these papers explicitly utilize the shadowing assumptions which have no constructions. To the best of our knowledge, up to now there have been less investigations of the random periodic Lipschitz shadowing of SDEs in the literature. Shadowing is still an interesting method for studying their random periodic dynamic behavior of SDEs.

It is well known that Lipschitz shadowing is the extension of classical shadowing, which is widely used in numerical 
analysis and computation. Therefore it is very meaningful to extend this definition to the stochastic periodic case, which is defined as $(\omega, L \delta)$-periodic Lipschitz shadowing orbits (RPLSO). Then we only need to construct some conditions such that the systems of SDEs possess RPLSO. In fact, these conditions can guarantee that the systems of SDEs have the stochastic periodic Lipschitz shadowing implicitly. Therefore, this provides an important method which is used in the proof of the existence of RPLSO, and it follows from stochastic calculus that the shadowing distance can be determined for any given $(\omega, \delta)$-pseudoperiodic orbit. This is the essence of the stochastic periodic Lipschitz shadowing which has been investigated from such practical point of view. And this brings great convenience to numerical analysis, so it can be an available and realistic method of estimating shadowing distance, that is, the maximum distance between an $(\omega, \delta)$ pseudoperiodic orbit and its corresponding nearest true random periodic orbit in mean-square sense.

Utilizing forward infinite horizon stochastic integral equations, we propose the finite-time random periodic Lipschitz shadowing theorem of SDEs. By random Romberg algorithm and random numerical computation, the shadowing distance is obtained. These results show that under some appropriate conditions the numerical approximative random periodic orbits of SDEs are close to the true ones and shadowing distance can be well estimated.

A more detailed outline of this paper is as follows. Section 2 deals with some preliminaries addressed to clarify the presentation of concepts and norms used later. Section 3 is devoted to the feasibility analysis of the finite-time $(\omega, L \delta)$-periodic Lipschitz shadowing. Section 4 presents the numerical analysis of shadowing distance which contains the detailed numerical implementation method and its convergence analysis. Illustrative numerical experiments using the well-known stochastic logistic equation for the main results are included in Section 5. Section 6 summarizes the conclusions of this article.

\section{Preliminaries}

Let $(\Omega, \mathscr{F}, \mathbb{P})$ be a Wiener space, $W(t)(t \in \mathbb{R})$ be a standard $k$ dimensional Wiener process, and $\left\{\mathscr{F}_{t}\right\}_{t \in \mathbb{R}}$ be its natural normal filtration. And $\Omega=\left\{\omega \in C\left(\mathbb{R}, \mathbb{R}^{k}\right): \omega(0)=0\right\}$ which means that the elements of $\Omega$ can be identified with paths of a Wiener process $\omega(t)=W_{t}(\omega)$. We consider a class of Ito SDEs of the form

$$
\begin{aligned}
& d X_{t}=-A X_{t} d t+f\left(t, X_{t}\right) d t+g(t) d W_{t}, \\
& \\
& X(0)=X_{0} \in \mathbb{R}^{d},
\end{aligned}
$$

where $X_{t}: \Omega \rightarrow \mathbb{R}^{d}, f: \mathbb{R} \times \mathbb{R}^{d} \rightarrow \mathbb{R}^{d}, g: \mathbb{R} \rightarrow \mathbb{R}^{d \times k}, A$ is a $d \times d$ hyperbolic matrix, whose real part of the eigenvalue is positive, and we define that $T_{t}=e^{-A t}$ is a pseudohyperbolic linear flow induced by $-A$; the initial value $X_{0}$ is independent of $\mathscr{F}_{0}$ and satisfies the inequality $\mathbb{E}\left|X_{0}\right|^{2}<\infty$.

2.1. Basic Assumptions and Notations. In this paper, we make the following assumptions which are made for the theoretical analysis.
Hypothesis 1 (see [6]). (i) The initial value $X_{0}$ is bounded; that is, $\left\|X_{0}\right\| \leq K$ for $K>0$.

(ii) Assume that the function $f: \mathbb{R} \times \mathbb{R}^{d} \rightarrow \mathbb{R}^{d}$ is continuous, measurable function, and the function $g: \mathbb{R} \rightarrow$ $\mathbb{R}^{d}$ is continuous too. Suppose that there exists a constant $\tau>0$ such that for $\forall t \in \mathbb{R}$ and $\forall X \in \mathbb{R}^{d}$,

$$
\begin{aligned}
f(t, X) & =f(t+\tau, X), \\
g(t) & =g(t+\tau) .
\end{aligned}
$$

(iii) The function $f$ is locally bounded, locally Lipschitz with respect to the second variable, and is a $C^{1}$ vector field on $\mathbb{R}^{d}$. That is, there exist positive constants $J, M$, and $K_{1}$ such that $\|f(t, 0)\| \leq J$ holds for $t \in \mathbb{R}$ and $\forall\|X\|,\|Y\| \leq M$; then

$$
\|f(t, X)-f(t, Y)\| \leq K_{1}\|X-Y\| .
$$

(iv) The function $g$ is globally bounded. That is, there exists a constant $K_{2}>0$ such that $|g(t)| \leq K_{2}$ holds for $t \in \mathbb{R}$.

We define

$$
\begin{aligned}
\theta: \mathbb{R} \times \Omega & \longrightarrow \Omega, \\
\theta^{t} \omega(s) & =W(t+s)-W(t),
\end{aligned}
$$

and $s \leq t, s \in \mathbb{R}, t \in \mathbb{R}$. By the conclusions in [2], SDE (1) generates a stochastic flow $\varphi: \mathbb{R} \times \mathbb{R} \times \Omega \times \mathbb{R}^{d} \rightarrow \mathbb{R}^{d}$ when the solution of SDE (1) exists uniquely, which is usually written as $\varphi(s, t, \omega, X):=\varphi(s, t, \omega) X$ on the metric dynamical systems $\left(\Omega, \mathscr{F}, \mathbb{P}, \theta^{t}\right)$. The stochastic flow $\varphi$ is given by

$$
\begin{aligned}
\varphi(s, t, \omega) X \\
=X+\int_{s}^{t}(-A \varphi(s, r, \omega) X+f(r, \varphi(s, r, \omega) X)) d r \\
\quad+\int_{s}^{t} g(\varphi(s, r, \omega) X, r) d W_{r}, \quad t \geq s .
\end{aligned}
$$

Remark 1. Here the expression $\varphi(s, t, \omega) X$ denotes a solution $\varphi$ of SDE (1) at the time $t$ which has the sample $\omega$ at the initial time $s$ and initial value $X$. And the random periodic solution is defined as below. If $U(t, \omega)$ is a random periodic solution of $\varphi(s, t, \omega) X$, then we obtain that

$$
\varphi(t, t+\tau, \omega) U(t, \omega)=U(t+\tau, \omega)=U\left(t, \theta^{\tau} \omega\right),
$$

for any $t \in \mathbb{R}^{+}$and $\omega \in \Omega$. Furthermore, in this paper, we only consider the case that $A$ is a deterministic matrix. Because it needs complex theory and tools, the random case $A(\omega)$ will be investigated in our future work.

Remark 2. It follows from the conclusions in $[2,12]$ that the definition of RDS is the extension of the definition of stochastic flow; that is, the latter is a particular case of the former. Therefore, some conclusions in [6] are also valid in this article.

We also utilize the notations as follows. 
(i) For any random vector $X=\left(X_{1}, X_{2}, \ldots, X_{d}\right) \in$ $L^{2}(\Omega)$, we define

$$
\begin{gathered}
\|X\|_{2}=\left[\int _ { \Omega } \left[\left|X_{1}(\omega)\right|^{2}+\left|X_{2}(\omega)\right|^{2}+\cdots\right.\right. \\
\left.\left.+\left|X_{d}(\omega)\right|^{2}\right] d \mathbb{P}(\omega)\right]^{1 / 2}<\infty .
\end{gathered}
$$

(ii) For a stochastic process $X(t, \omega)$ with $X_{t}(\omega) \in L^{2}(\Omega)$ and $t \in \mathbb{R}$, we define

$$
\|X(t, \omega)\|_{2}=\sup _{t \in \mathbb{R}}\left\|X_{t}(\omega)\right\|_{2}<\infty .
$$

(iii) The norm of random matrix is defined in the form of

$$
\|B\|_{L^{2}(\Omega)}=\left[\mathbb{E}\left(|B|^{2}\right)\right]^{1 / 2},
$$

where $B$ is a random matrix and $|\cdot|$ is the operator norm.

(iv) In the continuation the norm $\|\cdot\|_{2}$ and $\|\cdot\|_{L^{2}(\Omega)}$ are written as $\|\cdot\|$ unless otherwise stated.

2.2. Some Concepts and Remarks. We will extend the definitions of $(\omega, \delta)$-pseudoorbit and $(\omega, \varepsilon)$-shadowing in [6] to the random periodic case, which also referr to the definitions of pseudoperiodic orbit and periodic shadowing in $[7,13]$.

Definition 3. For a given positive number $\delta$, if there is a sequence of positive times $\left\{t_{k}\right\}_{k=0}^{N+1}, 0 \leq t_{0} \leq t_{1} \leq \cdots \leq \tau \leq$ $t_{N+1}, \tau$ and a sequence of random variables

$$
\left\{\left(Y_{k}\left(\theta^{t_{k}} \omega\right), \mathscr{F}_{t_{k}}\right)\right\}_{k=0}^{N},
$$

which means that $Y_{k}\left(\theta^{t_{k}} \omega\right)$ is $\mathscr{F}_{t_{k}}$-adapted for $k=0,1,2$, $\ldots, N$ and $\omega \in \Omega$, such that the following inequalities hold

$$
\begin{array}{r}
\left\|Y_{k+1}\left(\theta^{t_{k+1}} \omega\right)-\varphi\left(t_{k}, t_{k+1}, \theta^{t_{k}} \omega\right) Y_{k}\left(\theta^{t_{k}} \omega\right)\right\| \leq \delta, \\
k=0,1, \ldots, N-1, \\
\left\|Y_{N}\left(\theta^{t_{N}} \omega\right)-Y_{0}\left(\theta^{t_{0}} \omega\right)\right\| \leq \delta,
\end{array}
$$

then the random variables $\left\{\left(Y_{k}\left(\theta^{t_{k}} \omega\right), \mathscr{F}_{t_{k}}\right)\right\}_{k=0}^{N}$ are said to be a $(\omega, \delta)$-pseudorandom periodic orbit of SDE (1) in meansquare sense, where the inequality describes the random periodic property, and it is an extension of the corresponding definitions; we refer for the details to $[6,7,13]$.

Definition 4 (see [14]). For a given positive number $\delta_{0}>$ $0, L>0$, and any $(\omega, \delta)$-pseudorandom periodic orbit $\left\{\left(Y_{k}\left(\theta^{t_{k}} \omega\right), \mathscr{F}_{t_{k}}\right)\right\}_{k=0}^{N}\left(0<\delta \leq \delta_{0}\right)$ of SDE (1) with associated times $\left\{t_{k}\right\}_{k=0}^{N+1}$, if there is a sequence of times $\left\{h_{k}\right\}_{k=0}^{N+1}$, $h_{0} \leq h_{1} \leq \cdots \leq \tau \leq h_{N+1}$, and random variables $\left\{\left(X_{k}\left(\theta^{h_{k}} \omega\right), \mathscr{F}_{h_{k}}\right)\right\}_{k=0}^{N}$, such that the following inequalities hold

$$
\begin{aligned}
&\left\|Y_{k}\left(\theta^{t_{k}} \omega\right)-X_{k}\left(\theta^{h_{k}} \omega\right)\right\| \leq L \delta, \\
& 0 \leq t_{k}-h_{k} \leq L \delta:=\varepsilon, k=0,1, \ldots, N,
\end{aligned}
$$

and random variables $\left\{\left(X_{k}\left(\theta^{h_{k}} \omega\right), \mathscr{F}_{h_{k}}\right)\right\}_{k=0}^{N}$ are on the true orbits of SDE (1), that is

$$
\begin{gathered}
X_{k+1}\left(\theta^{h_{k+1}} \omega\right)=\varphi\left(h_{k}, h_{k+1}, \theta^{h_{k}} \omega\right) X_{k}\left(\theta^{h_{k}} \omega\right), \\
k=0,1,2, \ldots, N-1, \\
X_{0}\left(\theta^{h_{0}} \omega\right)=\varphi\left(h_{N}, h_{N+1}, \theta^{h_{N}} \omega\right) X_{N}\left(\theta^{h_{N}} \omega\right),
\end{gathered}
$$

then the $(\omega, \delta)$-pseudorandom periodic orbit $\left\{\left(Y_{k}\left(\theta^{t_{k}} \omega\right)\right.\right.$, $\left.\left.\mathscr{F}_{t_{k}}\right)\right\}_{k=0}^{N}$ is said to be $(\omega, L \delta)$-periodic Lipschitz shadowed by a true orbit of SDE (1) in mean-square sense.

Remark 5. As the $\sigma$-algebra $\mathscr{F}_{t}(t \geq 0)$ is nondecreasing, in order to guarantee the random variable $X_{k}\left(\theta^{h_{k}} \omega\right)(k=$ $0,1,2, \ldots, N)$ is $\mathscr{F}_{t_{k}}$-measurable, we need the shadowing condition $0 \leq t_{k}-h_{k} \leq \varepsilon$ instead of $\left|t_{k}-h_{k}\right| \leq \varepsilon$ for deterministic counterpart [2]. We choose a sequence of times $\left\{h_{k}\right\}_{k=0}^{N+1}=\left\{t_{k}\right\}_{k=0}^{N+1}$ in sequels.

Remark 6. $(\omega, L \delta)$-periodic Lipschitz shadowing is a special case of $(\omega, \delta)$-periodic shadowing; that is, the former obtains an explicit dependent relationship between the local error and the shadowing distance, but the latter does not.

\section{Feasibility Analysis of $(\omega, L \delta)$-Periodic Lipschitz Shadowing}

Theorem 7. Suppose that $A$ is a class of hyperbolic $d \times d$ matrix and the eigenvalues of $A$ are denoted by $\left\{\lambda_{j}, j=1,2, \ldots, d\right\}$ which satisfy $0<\operatorname{Re}\left(\lambda_{1}\right) \leq \operatorname{Re}\left(\lambda_{2}\right) \leq \cdots \leq \operatorname{Re}\left(\lambda_{d}\right)$. Let $\left\{\left(Y_{k}\left(\theta^{t_{k}} \omega\right), \mathscr{F}_{t_{k}}\right)\right\}_{k=0}^{N}$ be a bounded $(\omega, \delta)$-pseudorandom periodic orbit of $S D E(1), 0<\delta<\delta_{0}$. Moreover, suppose that SDE (1) satisfies Hypothesis 1 and the maximum of the locally Lipschitz constant of $f$ be $K_{1} \in\left[0, \sqrt{2} \operatorname{Re}\left(\lambda_{d}\right)\right)$ with respect to the $(\omega, \delta)$-pseudorandom periodic orbit of SDE (1) $\left\{\left(Y_{k}\left(\theta^{t_{k}} \omega\right), \mathscr{F}_{t_{k}}\right)\right\}_{k=0}^{N}$.

Then there exists a sequence of points $\left\{\left(X_{k}\left(\theta^{h_{k}} \omega\right), \mathscr{F}_{t_{N}}\right)\right\}_{k=0}^{N}$ and a constant $L>0$ such that the $(\omega, \delta)$-pseudorandom periodic orbit $\left\{\left(Y_{k}\left(\theta^{t_{k}} \omega\right), \mathscr{F}_{t_{k}}\right)\right\}_{k=0}^{N}$ is $(\omega, L \delta)$-periodic Lipschitz shadowed by a true orbit of SDE (1) which contains this sequence of points $\left\{\left(X_{k}\left(\theta^{h_{k}} \omega\right), \mathscr{F}_{t_{k}}\right)\right\}_{k=0}^{N}$ in mean-square sense, where times $h_{k}=t_{k}, k=0,1, \ldots, N+1$.

Proof. Firstly, for a given constant $\delta_{0}$ and a bounded $(\omega, \delta)$ pseudoperiodic orbit of SDE (1) $\left\{\left(Y_{k}\left(\theta^{t_{k}} \omega\right), \mathscr{F}_{t_{k}}\right)\right\}_{k=0}^{N}$, if we choose any $\delta \in\left(0, \delta_{0}\right)$ and an initial value $\bar{X}_{t_{0}}$ such that $\| \bar{X}_{t_{0}}-$ $Y_{0}\left(\theta^{t_{0}} \omega\right) \| \leq \delta$, then we can prove that $\operatorname{SDE}$ (1) with initial value $\bar{X}_{t_{0}}$ has a unique random periodic solution $Y(t, \omega)$ : $(-\infty,+\infty) \times \Omega \rightarrow \mathbb{R}^{d}$, and that $Y(t, \omega)$ is a solution of the forward infinite horizon integral equation

$$
\begin{aligned}
Y(t, \omega)= & \int_{-\infty}^{t} T_{t-r} f(r, Y(r, \omega)) d r \\
& +(\omega) \int_{-\infty}^{t} T_{t-r} g(r) d W_{r} .
\end{aligned}
$$


This claim is proved by a truncation procedure. As we have done in [3], we only need to take the limit as $J \rightarrow+\infty$. For each $J \geq 1$ and $J \in \mathbb{Z}$, define the truncation function

$$
f_{J}(t, X)= \begin{cases}f(t, X), & \text { if }\|X\| \leq J, \\ f\left(t, \frac{J X}{\|X\|}\right), & \text { if }\|X\|>J .\end{cases}
$$

Then it follows from Theorem 3.3 in [5] that there is a unique random periodic solution $X_{t}^{J}:=Y^{J}(t, \omega)$ to the equation

$$
\begin{aligned}
d X_{t}^{J}=-A X_{t}^{J} d t+f_{J}\left(t, X_{t}^{J}\right) d t+g(t) d W_{t}, & \\
& X^{J}(0)=X_{0} \in \mathbb{R}^{d} .
\end{aligned}
$$

Define the stopping time

$$
\tau_{J}=t_{N+1} \wedge \inf \left\{t \in\left[0, t_{N+1}\right],\left\|X_{t}^{J}\right\| \geq J\right\} .
$$

We can show that

$$
X_{t}^{J}=X_{t}^{J+1}, \quad \text { if } 0 \leq t \leq \tau_{J} .
$$

This implies that $\tau_{J}$ is increasing. Then we can use the linear growth condition to prove that for almost all $\omega \in \Omega$, there exists an integer $J_{0}=J_{0}(\omega)$ such that $\tau_{J}=t_{N+1}$ whenever $J \geq J_{0}$.

Now we define $X_{t}$ by

$$
X_{t}=X_{t}^{J_{0}}, \quad t \in\left[0, t_{N+1}\right]
$$

It follows from (19) that we have $X_{\left(t \wedge \tau_{J}\right)}=X_{\left(t \wedge \tau_{J}\right)}^{J}$, and by (17) we obtain that

$$
\begin{aligned}
d X_{\left(t \wedge \tau_{J}\right)}^{J}= & -A X_{\left(t \wedge \tau_{J}\right)}^{J} d t+f_{J}\left(t, X_{\left(t \wedge \tau_{J}\right)}^{J}\right) d t \\
& +g(t) d W_{t} .
\end{aligned}
$$

Let $J \rightarrow \infty$; we see that $X_{t}$ is a solution of SDE (1). Therefore, this proved the existence of the true orbit of SDE (1) with proper modified initial conditions.

Secondly, we only need to prove that a $(\omega, \delta)$-pseudoperiodic orbit $\left\{\left(Y_{k}\left(\theta^{t_{k}} \omega\right), \mathscr{F}_{t_{k}}\right)\right\}_{k=0}^{N}$ is $(\omega, L \delta)$-periodic Lipschitz shadowed by this sequence of points $\left\{\left(X_{k}\left(\theta^{h_{k}} \omega\right)\right.\right.$, $\left.\left.\mathscr{F}_{t_{k}}\right)\right\}_{k=0}^{N}$, which lie on a true orbit of SDE (1); that is, we only need to prove that (14) holds.

It follows from the above that the random periodic solution $Y(t, \omega)$ of SDE (1) exists and its expression can be provided as (15). In the finite-time interval $\left[0, t_{N+1}\right]$ we can choose $\delta \in\left(0, \delta_{0}\right)$, and the distance $\Delta_{k}$ between the $(\omega, \delta)$ pseudoperiodic orbit $\left\{\left(Y_{k}\left(\theta^{t_{k}} \omega\right), \mathscr{F}_{t_{k}}\right)\right\}_{k=0}^{N}$ and the points on random periodic solution $Y(t, \omega)$ at the time $\left\{t_{k}\right\}_{k=0}^{N+1}$ is a finite constant $\Delta_{k}$. With this method we can choose

$$
L:=\max _{0 \leq k \leq N}\left\{\frac{\Delta_{k}}{\delta}\right\}
$$

as the Lipschitz constant and

$$
\Delta=\max _{0 \leq k \leq N}\left\{\Delta_{k}\right\}
$$

as the radius of $(\omega, \delta)$-pseudoperiodic orbit's neighbourhood. Therefore, the conclusion is immediate from Definition 4. This completes the proof of Theorem 7 .

Remark 8. The difference from [5] is that the space is $\mathbb{R}^{d}$, not a subspace $B \subset \mathbb{R}^{d}$.

\section{Numerical Implementation of Shadowing Distance}

4.1. A Detailed Implementation Method of Shadowing Distance. We show the numerical method in detail, which we use for the approximation of shadowing distance, and this method consists of three steps as follows.

Step 1. Utilizing the one-step numerical scheme (EulerMaruyama (EM) scheme, Milstein scheme [15]) to solve the following equation from $t_{k}$ to $t_{k+1}$ with the initial values $X(0)=X_{0}$,

$$
d X_{t}=-A X_{t} d t+f\left(t, X_{t}\right) d t+g(t) d W_{t}
$$

we obtain the approximations of $X_{k+1}$, that is, $(\omega, \delta)$ pseudoperiodic orbit of SDE (1),

$$
\begin{aligned}
X_{k+1}= & X_{k}+\left(-A X_{k}+f\left(t_{k}, X_{k}\right)\right)\left(t_{k+1}-t_{k}\right) \\
& +g\left(t_{k}\right) \Delta W_{k} .
\end{aligned}
$$

Step 2. It follows from Theorem 7 that the forward infinite horizon integral equation (15) is the random periodic solution of SDE (1) with new initial conditions which is chosen as Section 3. It follows from Theorem 3.4 in [5] that the random periodic solution (15) is mean-square uniformly asymptotically stable.

In order to make this article self-contained we outline the numerical method which is used for approximating the random periodic solution. The reader is referred to the paper [5] for a more detailed description. Therefore, numerical implementation method of the random periodic orbits is described as follows.

Firstly, we need to obtain the initial value for the sake of the approximation. It follows from (15) that we get

$$
\begin{aligned}
Y(0, \omega)= & \int_{-\infty}^{0} T_{-r} f(r, Y(r, \omega)) d r \\
& +(\omega) \int_{-\infty}^{0} T_{-r} g(r) d W_{r}
\end{aligned}
$$

For any given presupposed error tolerance $\widehat{\delta} \in(0, \Delta t]$, if $s^{\prime}<$ 0 is chosen such that the following inequality holds

$$
\begin{gathered}
\| Y(0, \omega)-\int_{s^{\prime}}^{0} T_{-r} f(r, Y(r, \omega)) d r \\
-(\omega) \int_{s^{\prime}}^{0} T_{-r} g(r) d W_{r} \| \leq \widehat{\delta},
\end{gathered}
$$


then the Ito integral $\bar{Y}(0, \omega)$ can be used to approximate the improper integral (26), where

$$
\begin{aligned}
\bar{Y}(0, \omega)= & \int_{s^{\prime}}^{0} T_{-r} f(r, \bar{Y}(r, \omega)) d r \\
& +(\omega) \int_{s^{\prime}}^{0} T_{-r} g(r) d W_{r},
\end{aligned}
$$

and $\Delta t:=t_{N+1} / N^{\prime}$. Therefore, $\bar{Y}(0, \omega)$ can be chosen as the approximating initial value in the given presupposed error tolerance.

Secondly, we need to obtain the approximation of the improper integral (15). Therefore the improper integral (15) in the finite-time interval $\left[0, t_{N+1}\right]$ can be approximated by the Ito integral (29)

$$
\begin{aligned}
\tilde{Y}\left(t_{N+1}, \omega\right)= & \int_{0}^{t_{N+1}} T_{t-r} f(r, \tilde{Y}(r, \omega)) d r \\
& +(\omega) \int_{0}^{t_{N+1}} T_{t-r} g(r) d W_{r},
\end{aligned}
$$

with initial value $\bar{Y}(0, \omega)$ at the time $t=0$. It follows from (29), pullback theory, and the construction of the random periodic orbits that $\widetilde{Y}\left(t_{N+1}, \omega\right)$ is $\omega$-wise, too. Here, pullback theory is pullback random analysis theory which is shown as [1] in detail. Furthermore, $\widetilde{Y}\left(t_{N+1}, \omega\right)$ and $\bar{Y}(0, \omega)$ have the same sample $\omega$ at the time $t=0$.

Remark 9. By means of reselecting the corresponding starting time and $s^{\prime}$, we can simulate a random periodic solution in an arbitrary finite-time interval with any given presupposed error tolerance.

Finally, in order to improve the accuracy of the integral, the random Romberg algorithm is applied to $(29)$ and $\bar{Y}(0, \omega)$. The method applied to (29) is shown as follows briefly.

Let $\widetilde{Y}_{n}(t, \omega)$ be the approximation of $\widetilde{Y}(0, n \Delta t, \omega)$; then we can obtain

$$
\begin{aligned}
\widetilde{Y}_{n}(t, \omega)= & \int_{0}^{n \Delta t} T_{n \Delta t-r} f\left(r, \widetilde{Y}_{n-1}(r, \omega)\right) d r \\
& +(\omega) \int_{0}^{n \Delta t} T_{n \Delta t-r} g(r) d W_{r},
\end{aligned}
$$

where $n=1, \ldots, N^{\prime}$ and $\widetilde{Y}_{N^{\prime}}(t, \omega)$ is the numerical approximation of $\widetilde{Y}\left(t_{N+1}, \omega\right)$.

For any given presupposed error tolerance $\widetilde{\delta} \in[0, \widehat{\delta}]$, if the following inequality holds

$$
\left\|R_{j, j-1}-R_{j j}\right\| \leq \widetilde{\delta},
$$

the computation of the random Romberg algorithm is ended and $R_{j j}$ is viewed as the approximation of (30). That is,

$$
\widetilde{Y}_{n}(t, \omega)=R_{j j},
$$

where $R_{j, j-1}$ and $R_{j j}$ are obtained by the implementation of the random Romberg algorithm; this algorithm is shown in detail in [5].
Step 3. It follows from Steps 1 and 2 and Theorem 7 that we can obtain the approximation of shadowing distance and the Lipschitz constant of Lipschitz shadowing as follows:

$$
\begin{aligned}
\Delta & =\max _{n}\left\{\left\|X_{n}-\widetilde{Y}_{n}(t, \omega)\right\|+\widehat{\delta}\right\}, \\
L & :=\frac{\Delta}{\delta} .
\end{aligned}
$$

4.2. Convergence Analysis. We can divide the time interval $\left[0, t_{N+1}\right]$ into $N^{\prime}$ subintervals with the length $\Delta t$. Then we obtain the exact solution of SDE (1) in $\left[0, t_{N+1}\right]$ as follows:

$$
\begin{aligned}
\check{Y}_{n}(t, \omega)= & \int_{0}^{n \Delta t} e^{-A(n \Delta t-r)} f\left(r, \breve{Y}_{n}(r, \omega)\right) d r \\
& +(\omega) \int_{0}^{n \Delta t} e^{-A(n \Delta t-r)} g(r) d W_{r} .
\end{aligned}
$$

Therefore the theoretical shadowing distance is shown as follows:

$$
\bar{\Delta}=\max _{n}\left\{\left\|X_{n}-\check{Y}_{n}(t, \omega)\right\|+\widehat{\delta}\right\} .
$$

We will show that the shadowing distance (33) is bounded. And the numerical approximation (33) to shadowing distance is mean-square convergent to the theoretical result (35).

Theorem 10. Suppose that $X_{0} \in L^{2}(\Omega), S D E$ (1) satisfy the condition of Theorem 7 , then the shadowing distance (33) is bounded, and the numerical approximation (33) to shadowing distance is mean-square convergent to the theoretical result (35) by the random Romberg algorithm.

Proof. First and foremost, it follows from (30) that

$$
\begin{aligned}
& \mathbb{E}\left|X_{n+1}-\widetilde{Y}_{n+1}(t, \omega)\right|^{2} \leq \mathbb{E} \mid\left(\mathbb{\square}-\left(t_{n+1}-t_{n}\right) A\right) X_{n} \\
& -\int_{0}^{(n+1) \Delta t}\left(f\left(r, X_{n}\right)\right. \\
& \left.-T_{(n+1) \Delta t-r} f\left(r, \widetilde{Y}_{n}(r, \omega)\right)\right) d r-(\omega) \\
& \left.\quad \cdot \int_{0}^{(n+1) \Delta t}\left(1-T_{(n+1) \Delta t-r}\right) g(r) d W_{r}\right|^{2} \leq I_{1}+I_{2} \\
& \quad+I_{3},
\end{aligned}
$$

where

$$
\begin{aligned}
I_{1} & =3 \mathbb{E}\left|\left(\mathbb{\square}-\left(t_{n+1}-t_{n}\right) A\right) X_{n}\right|^{2}, \\
I_{2} & =3 \mathbb{E} \mid \int_{0}^{(n+1) \Delta t}\left(f\left(r, X_{n}\right)\right. \\
& \left.-T_{(n+1) \Delta t-r} f\left(r, \widetilde{Y}_{n}(r, \omega)\right)\right)\left.d r\right|^{2}, \\
I_{3} & =3 \mathbb{E}\left|(\omega) \int_{0}^{(n+1) \Delta t}\left(1-T_{(n+1) \Delta t-r}\right) g(r) d W_{r}\right|^{2} .
\end{aligned}
$$


Utilizing the boundedness of $X_{0}$, there exists a positive constant $M_{1}$ such that $I_{1} \leq M_{1} \Delta t$. By the conclusion, we obtain that $0<T_{(n+1) \Delta t-r}<1$. Then it follows from the integral property and the Lipschitz condition of the function $f$ that

$$
\begin{aligned}
I_{2} & \leq 3 \mathbb{E}\left|\int_{0}^{(n+1) \Delta t}\left(f\left(r, X_{n}\right)-f\left(r, \widetilde{Y}_{n}(r, \omega)\right)\right) d r\right|^{2} \\
& \leq 3 K_{1}^{2} \int_{0}^{(n+1) \Delta t} \mathbb{E}\left|X_{n}-\widetilde{Y}_{n}(r, \omega)\right|^{2} d r .
\end{aligned}
$$

It follows from Ito isometry and the boundness condition of the function $g$ that

$$
I_{3} \leq 3 \int_{0}^{(n+1) \Delta t} \mathbb{E}|g(r)|^{2} d r \leq 3 K_{2}^{2}(n+1) \Delta t .
$$

Therefore by the Gronwall inequality, there exists a number $M_{2}$ such that (36) implies that

$$
\Delta_{n}=\left[\mathbb{E}\left|X_{n}-\widetilde{Y}_{n}(t, \omega)\right|^{2}\right]^{1 / 2}+\widehat{\delta} \leq M_{2},
$$

where $M_{2}=\sqrt{\left(M_{1}+3 K_{2}^{2} n\right) \Delta t \cdot \exp \left(3 K_{1}^{2} n \Delta t\right)}$.

By the conclusions (40), the first conclusion of Theorem 10 holds; that is, the shadowing distance $\Delta_{n}$ is bounded.

Secondly, from the expression of $\widetilde{Y}_{n}(t, \omega)$, we obtain

$$
\begin{aligned}
\tilde{Y}_{n}(t, \omega)= & \int_{0}^{n \Delta t} e^{-A(n \Delta t-r)} f\left(r, \tilde{Y}_{n-1}(r, \omega)\right) d r \\
& +(\omega) \int_{0}^{n \Delta t} e^{-A(n \Delta t-r)} g(r) d W_{r} .
\end{aligned}
$$

Then it implies that

$$
\mathbb{E}|\Delta-\bar{\Delta}|^{2} \leq \max _{n}\left\{\mathbb{E}\left|\check{Y}_{n}(t, \omega)-\widetilde{Y}_{n}(t, \omega)\right|^{2}\right\} .
$$

We let

$$
I_{4}:=\mathbb{E}\left|\breve{Y}_{n}(t, \omega)-\widetilde{Y}_{n}(t, \omega)\right|^{2},
$$

and we obtain that

$$
\begin{aligned}
I_{4} & =\mathbb{E}\left[\int _ { 0 } ^ { n \Delta t } e ^ { - A ( n \Delta t - r ) } \left[f\left(r, \breve{Y}_{n}(r, \omega)\right)\right.\right. \\
& \left.\left.-f\left(r, \widetilde{Y}_{n-1}(r, \omega)\right)\right] d r\right]^{2} .
\end{aligned}
$$

It follows from the Cauchy-Schwarz inequality that

$$
\begin{aligned}
I_{4} \leq & \int_{0}^{n \Delta t} e^{-2 A(n \Delta t-r)} d r \\
& \cdot \int_{0}^{n \Delta t} K_{1}^{2} \mathbb{E}\left|\breve{Y}_{n}(r, \omega)-\widetilde{Y}_{n-1}(r, \omega)\right|^{2} d r \\
\leq & K_{3} K_{1}^{2} \int_{0}^{n \Delta t} \mathbb{E}\left|\widetilde{Y}_{n}(r, \omega)-\widetilde{Y}_{n-1}(r, \omega)\right|^{2} d r,
\end{aligned}
$$

where $K_{3}=\int_{0}^{t_{N+1}} e^{-2 A(t-r)} d r$. that

Then the fact that $(a+b)^{2} \leq 2 a^{2}+2 b^{2}, a, b \in \mathbb{R}$, implies

$$
\begin{aligned}
I_{4} & \leq 2 K_{3} K_{1}^{2} \int_{0}^{n \Delta t}\left[\mathbb{E}\left|\widetilde{Y}_{n}(r, \omega)-\widetilde{Y}_{n}(r, \omega)\right|^{2}\right. \\
& \left.+\mathbb{E}\left|\widetilde{Y}_{n}(r, \omega)-\widetilde{Y}_{n-1}(r, \omega)\right|^{2}\right] d r .
\end{aligned}
$$

By the random Romberg algorithm in Section 4.1, we obtain

$$
\begin{aligned}
I_{4} \leq & 2 K_{3} \widehat{\delta}^{2} K_{1}^{2} t \\
& +2 K_{3} K_{1}^{2} \int_{0}^{n \Delta t} \mathbb{E}\left|\check{Y}_{n}(r, \omega)-\widetilde{Y}_{n}(r, \omega)\right|^{2} d r .
\end{aligned}
$$

It follows from the Gronwall inequality that there exists a number $M_{3}$ such that

$$
\left\|\check{Y}_{n}(t, \omega)-\widetilde{Y}_{n}(t, \omega)\right\| \leq M_{3},
$$

where

$$
M_{3}=\sqrt{\frac{2 K_{3} K_{1}^{2} t_{N+1}^{3}}{n^{2}} \cdot \exp \left(2 K_{3} K_{1}^{2} t_{N+1}\right)} .
$$

By the fact that $M_{3}$ tends to zero as $n \rightarrow+\infty$, we obtain

$$
\lim _{n \rightarrow+\infty}\left\|\widetilde{Y}_{n}(t, \omega)-\widetilde{Y}_{n}(t, \omega)\right\|=0 .
$$

That is,

$$
\lim _{n \rightarrow+\infty}\|\Delta-\bar{\Delta}\|=0 .
$$

Therefore, it is mean-square convergent. This proof is finished.

\section{Numerical Experiments}

5.1. Experimental Preparation. Assume that we are working in a one-dimensional space of real numbers and consider the following stochastic logistic equation:

$$
\begin{array}{r}
d X_{t}=-X_{t} d t+\left(5 X_{t}-4 X_{t}^{2}\right) d t+\mu \sin t d W_{t}, \\
X(0)=X_{0} ;
\end{array}
$$

that is,

$$
\begin{aligned}
A & =1, \\
f\left(t, X_{t}\right) & =5 X_{t}-4 X_{t}^{2}, \\
g(t) & =\mu \sin t .
\end{aligned}
$$

It follows from Theorem 7 that there exists random periodic solutions with period $\tau=2 \pi$ of SDE (52). If we choose $\widehat{\delta}=0.01, s^{\prime}$ is equal to -30 so that the inequality (27) holds. It follows from the results in Section 4.1 that we can obtain the numerical approximations in the presupposed initial error tolerance.

As shown in [5], to get the Brownian trajectory for the negative time, we construct the positive time path and reflect 

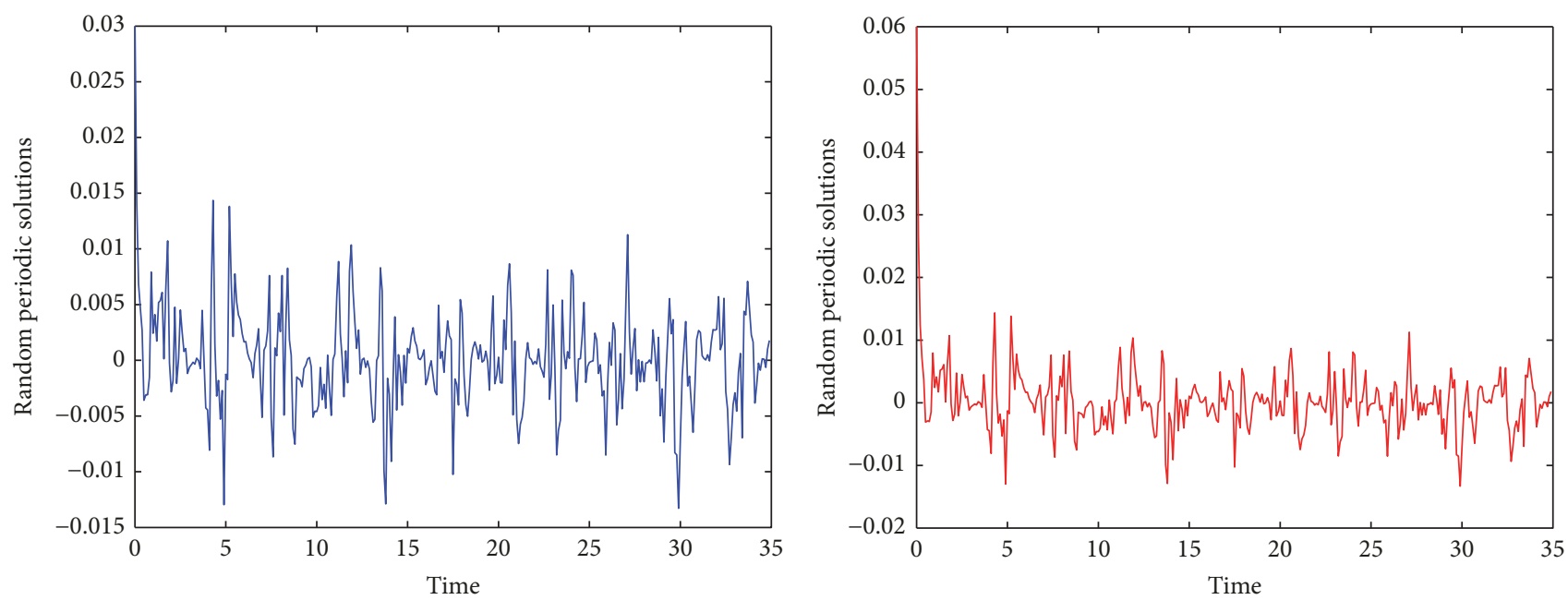

Figure 1: Random periodic solutions with the starting points $X_{0}=0.03$ and $X_{0}=0.06 ; \mu=2$.

it against point zero. We will run the simulation with the following meshes $[8,15]$ :

$$
\begin{aligned}
s^{\prime} & =-30, \\
t & =35, \\
\Delta t & =0.1, \\
N+1 & =350
\end{aligned}
$$

to construct a random periodic solution. We generate Brownian trajectories in the following way:

$$
\begin{aligned}
W_{0} & =0, \\
W_{(i+1) \Delta t} & =W_{i \Delta t}+\psi_{i+1},
\end{aligned}
$$

where $\psi_{i}=N(0, \sqrt{\Delta t}), i=1,2, \ldots, N+1$.

First and foremost, utilizing Theorem 7 and random Romberg algorithm we obtain the graphs for numerical approximations to random periodic solutions in the time interval $[0,35]$ as Figure 1 . As we see there exists random periodic phenomena with period $\tau=2 \pi$ with different starting points $X_{0}=0.03$ and $X_{0}=0.06$ at the time $t=0$.

Secondly, we need to verify the existence of random periodic orbits nearby a numerically computed $(\omega, \delta)$ pseudoperiodic orbit of SDE (52).

Utilizing the one-step numerical scheme (EM scheme [15]) to solve $\operatorname{SDE}(52)$ with the initial value $X_{0}=Y_{0}\left(\theta^{t_{0}} \omega\right)$, we obtain

$$
\begin{aligned}
X_{k+1}= & X_{k}+\left(-X_{k}+\left(5 X_{k}-4 X_{k}^{2}\right)\right) \Delta t_{k} \\
& +\mu \sin t_{k} \Delta W_{k}, \quad X(0)=X_{0},
\end{aligned}
$$

and then we obtain a numerically computed $(\omega, \delta)$ pseudoperiodic orbit of SDE (52).

Figure 2, whose starting points are $X_{0}=0.03$ and $\mu=2$, also reflects the fact that the true orbit is random periodic, and as we move forward in time, this true random periodic

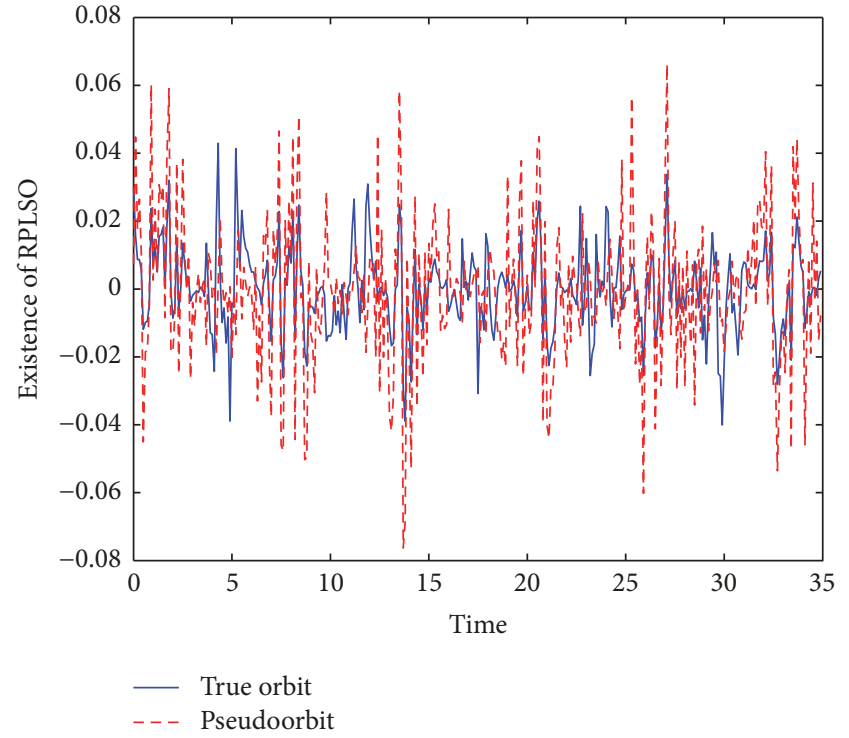

FIgURE 2: Existence of RPLSO.

orbit lies in the appropriate neighbourhood of the $(\omega, \delta)$ pseudoperiodic orbit of SDE (52). This shows that there exists $(\omega, L \delta)$-periodic Lipschitz shadowing in the systems of SDE (52). Meanwhile, we should pay attention to the fact that the relative position between the $(\omega, \delta)$-pseudoperiodic orbit and its $(\omega, L \delta)$-periodic Lipschitz shadowing orbit seems a little far away in some time. And this phenomenon is reflected in Figure 2; that is, the red line and the blue line are sometimes close, and sometimes far away. The reason is that the noise dumped into the systems constantly, and the random periodic solutions are stochastic processes which depend on every $\omega \epsilon$ $\Omega$. These results confirm the existence of RPLSO.

5.2. Numerical Results. This section will provide numerical experiments to compute the shadowing distance of SDE (52).

Firstly, in order to show the influence of noise on the $(\omega, \delta)$-pseudoperiodic orbit, we choose various sizes of noise, 

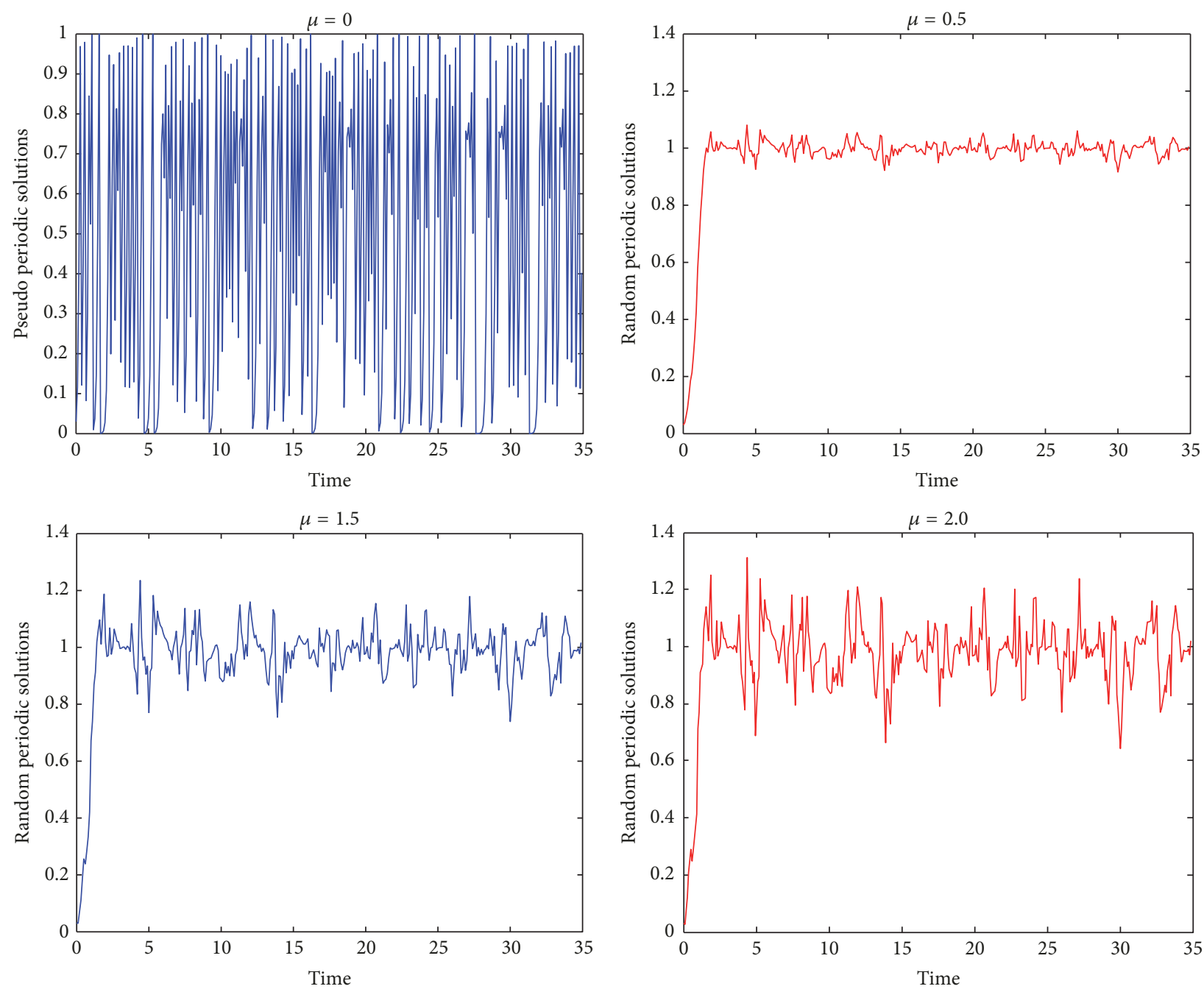

FIgURE 3: Pseudoorbits for different sizes of noise: $\mu=0, \mu=0.5, \mu=1.5$, and $\mu=2.0$.

such as $\mu=0$ (the deterministic case), $\mu=0.5, \mu=1.5$, and $\mu=2.0$. And we take the temporal step size $\Delta t=0.1$ and $N+1=350$. Taking the numerical solution as an example, Figure 3 shows the perturbation of the $(\omega, \delta)$-pseudoperiodic orbits of SDE (52) corresponding to differential scales of noise. It shows that the perturbation of the $(\omega, \delta)$ pseudoperiodic orbits becomes much more serious both in $x$ and in $y$ directions due to the increase in the scale of the noise when $\mu>0$.

Secondly, we focus on numerically performing the shadowing distance $\varepsilon$ shown as (33). The presupposed error tolerance $\widehat{\delta}$ is chosen as the step size $\Delta t$. The local error $\delta$ is approximately determined by the numerical scheme.

Table 1 presents the numerical results, where $\delta$ is the local error and $\varepsilon$ is the shadowing distance. It shows the existence of RPLSO and the effectiveness of the numerical method. Because we choose $\left\{h_{k}\right\}_{k=0}^{N+1}=\left\{t_{k}\right\}_{k=0}^{N+1}$, that is, we do not consider the reparameterization of time, the values of shadowing distance $\varepsilon$ are not small with respect to the local error $\delta$.

Figure 4 presents the shadowing distances of the $(\omega, \delta)$ pseudoperiodic orbits by the numerical computation with different error tolerance $\widehat{\delta}$. The first two figures are about the error tolerance $\widehat{\delta}=0.1$, and the other two figures are about the error tolerance $\widehat{\delta}=0.05$. In order to show the detailed case which is shown in Table 1, we only take the iterative step $N+1=350$. And the curve of the discrete shadowing orbits shows the oscillating property of the $(\omega, \delta)$-pseudoperiodic orbits. It also indicates the relevance between the discrete shadowing orbits and the $(\omega, \delta)$-pseudoperiodic orbits. This phenomena mean that numerical experiment consists with the theory result of Theorem 7.

As can be seen from these numerical results, there is an explicit dependent relationship between the shadowing distance and the local error, and there exists a true random periodic orbit in the appropriate neighbourhood of a given $(\omega, \delta)$-pseudoperiodic orbit of SDE (52). The numerical behavior of the system indeed reflects its real dynamical behavior.

Finally, to check the convergence of numerical approximations to the shadowing distance, we plot the curves from different starting points at the time $t=0$ in the same graph. As we see from Figure 5, whose starting points are $X_{0}=0.33$ 

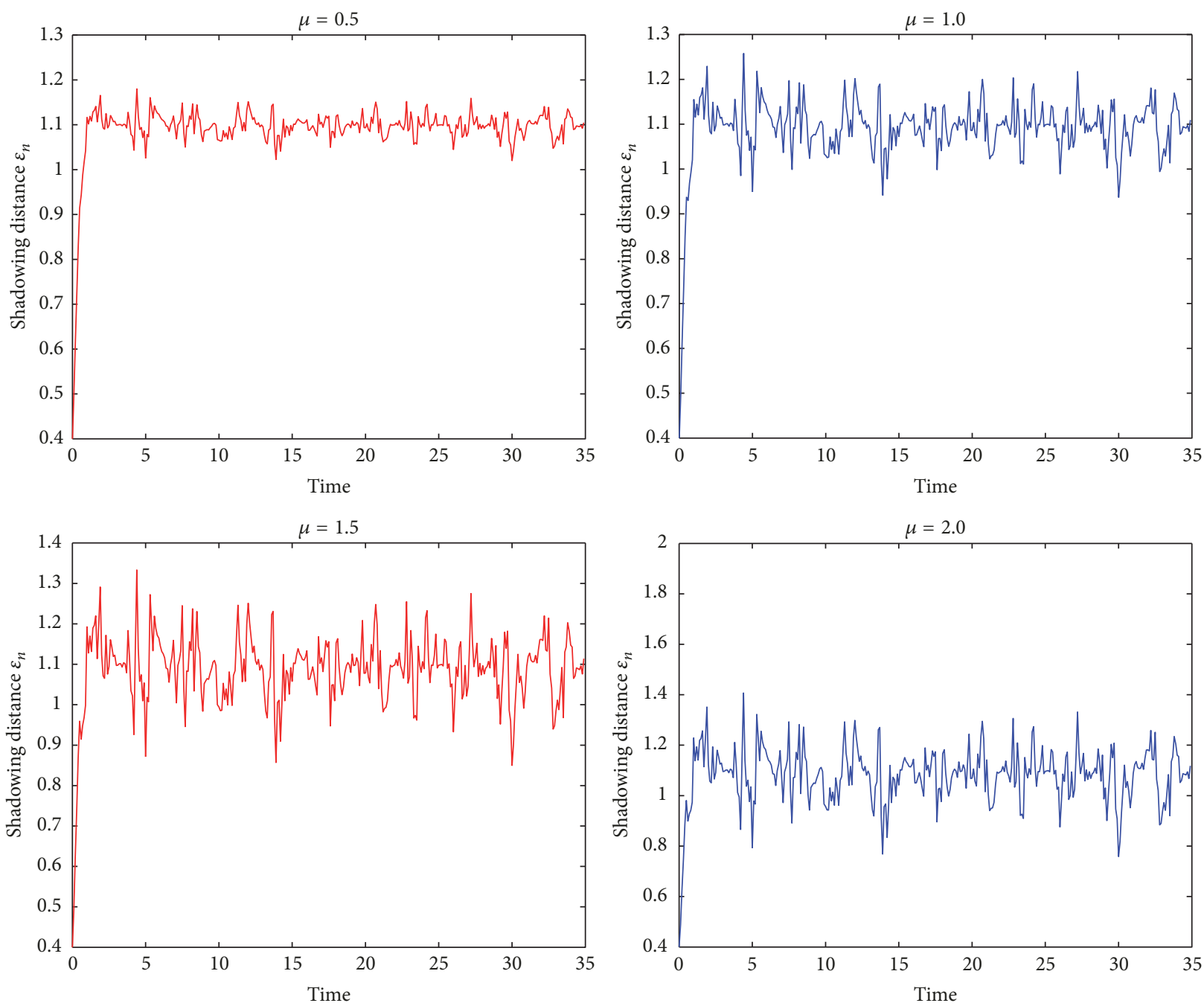

FIgURE 4: Zoom in parts showing the results in Table 1 for different error tolerance $\widehat{\delta}$ and noises.

TABLE 1: Summaries of the parameters for stochastic logistic equation.

\begin{tabular}{lcccc}
\hline EM & $\widehat{\delta}=0.1$ & $T=35.0$ & $\widehat{\delta}=0.05$ & \multicolumn{1}{c}{$T$} \\
$\mu$ & $\delta$ & $L \delta$ & 0.2236 & 17.5 \\
\hline 0.5 & 0.3162 & 1.1800 & 0.2236 & 1.0148 \\
1.0 & 0.3162 & 1.2578 & 0.2236 & 1.2097 \\
1.5 & 0.3162 & 1.3334 & 0.2236 & 1.2600 \\
2.0 & 0.3162 & 1.4070 & & \\
\hline
\end{tabular}

and $X_{0}=0.06$, respectively, as time progresses, the trajectories become asymptotically close. This also reflects the fact that whatever the starting points we choose, as we move forward in time, the shadowing distance $\varepsilon_{n}$ arrives at the exact trajectories which depend on different $\omega \in \Omega$; that is, the shadowing distance $\varepsilon_{n}$ is a stochastic process and different for every $\omega \in \Omega$, and its upper bound exists. These confirm that the numerical methods are efficient.

\section{Conclusion}

Finally, conclusions and future work are summarized. In this paper, the main result is $(\omega, L \delta)$-periodic Lipschitz shadowing of a class of stochastic differential equations. The methods shown in this paper focus on the possibility of $(\omega, L \delta)$-periodic Lipschitz shadowing. The results show that the methods are effective and the numerical results 


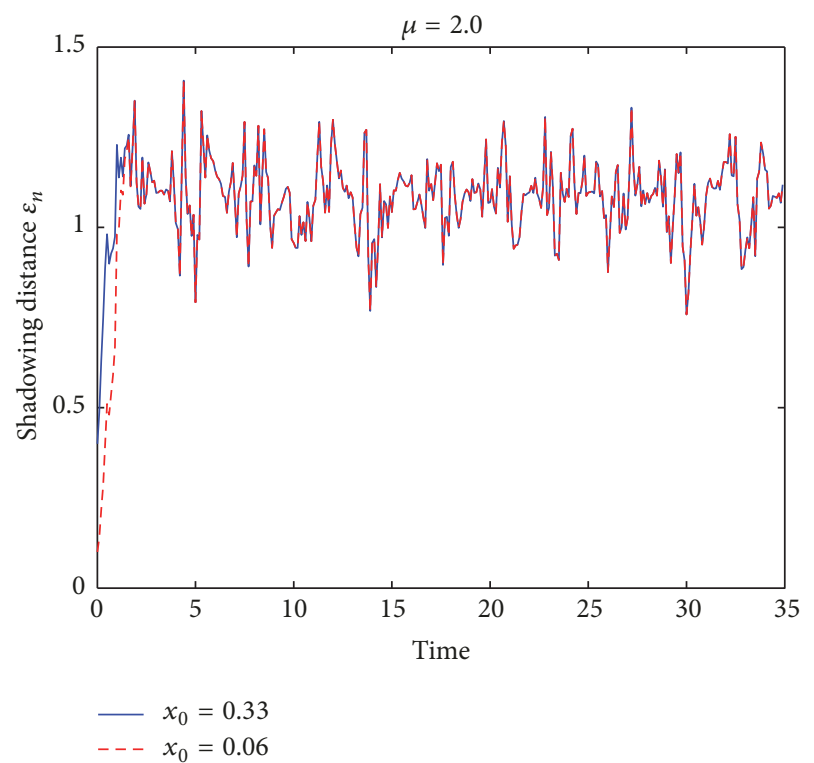

FIGURE 5: Convergence of the shadowing distance $\varepsilon_{n}$ with different starting points.

are performed and match the results of theoretical analysis. Although some progress is made, more simple and practical methods will be shown in our further work.

\section{Conflicts of Interest}

The authors declare that there are no conflicts of interest regarding the publication of this paper.

\section{Acknowledgments}

The authors would like to express their gratitude to Mr. Jialin Hong for his discussion. This work is supported by the Science Research Projection of the Education Department of Fujian Province, no. JAT160182, and the Natural Science Foundation of Fujian Province, no. 2015J01019.

\section{References}

[1] C. Feng, H. Zhao, and B. Zhou, "Pathwise random periodic solutions of stochastic differential equations," Journal of Differential Equations, vol. 251, no. 1, pp. 119-149, 2011.

[2] L. Arnold, Random Dynamical Systems, Springer, 2nd edition, 2003.

[3] X. Mao, Stochastic Differential Equations and Applications, Horwood, 2nd edition, 2008.

[4] B. F. Liu, Y. L. Han, and X. D. Sun, "Square-mean almost periodic solutions for a class of stochastic integro-differential equations," Journal of Jilin University. Science Edition, vol. 51, no. 3, pp. 393-397, 2013.

[5] Q. Zhan, "Mean-square numerical approximations to random periodic solutions of stochastic differential equations," Advances in Difference Equations, vol. 2015, no. 1, article no. 292, 2015.

[6] Q. Zhan, "Shadowing orbits of stochastic differential equations," Journal of Nonlinear Sciences and Applications, vol. 9, no. 5, pp. 2006-2018, 2016.
[7] Q. Zhan and Y. Li, Numerical Random Periodic Shadowing Orbits of A Class of Stochastic Differential Equations, "Dynamical Systems-Analytical and Computational Techniques", InTech, 2017.

[8] P. Wang, "A-stable Runge-Kutta methods for stiff stochastic differential equations with multiplicative noise," Computational and Applied Mathematics, 1 page, 2014, 20.

[9] T. Wang, "Optimal point-wise error estimate of a compact difference scheme for the coupled Gross-Pitaevskii equations in one dimension," Journal of Scientific Computing, vol. 59, no. 1, pp. 158-186, 2014.

[10] D. Todorov, Stochastic shadowing and stochastic stability, https://arxiv.org/abs/1411.7604.

[11] A. Fakhari and A. Golmakani, "Shadowing properties of random hyperbolic sets," International Journal of Mathematics, vol. 23, no. 6, Article ID 1250025, 2012.

[12] H. Kunita, Stochastic flows and stochastic differential equations, vol. 24 of Cambridge Studies in Advanced Mathematics, Cambridge University Press, Cambridge, 1997.

[13] K. J. Palmer, Shadowing in Dynamical Systems. Theory and Applications, Kluwer Academic, Dordrecht, The Netherlands, 2000.

[14] S. Y. Pilyugin, Shadowing in Dynamical Systems, vol. 1706 of Lecture Notes in Mathematics, Springer, Berlin, Germany, 1999.

[15] G. N. Milstein, Numerical Integration of Stochastic Differential Equations, vol. 313 of Mathematics and its Applications, Kluwer Academic Publishers Group, Dordrecht, The Netherlands, 1995. 


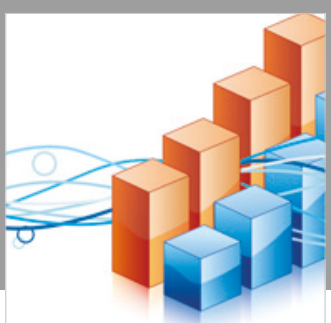

Advances in

Operations Research

\section{-n-m}
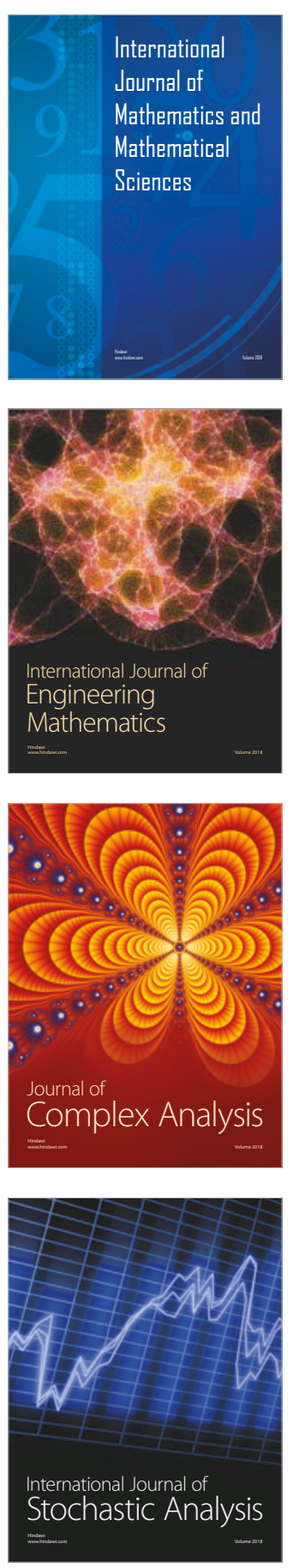
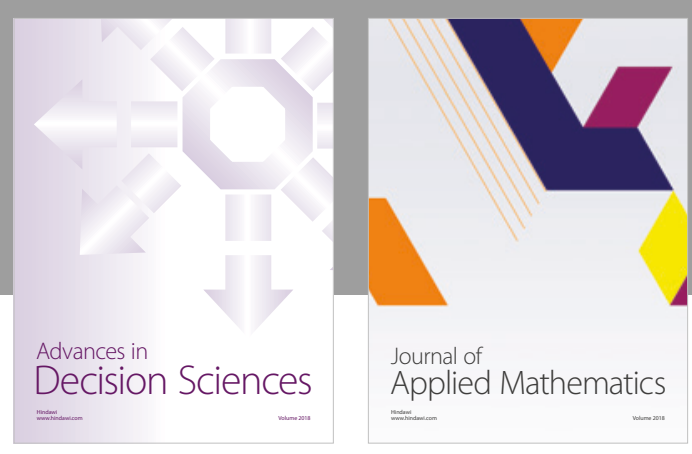

Journal of

Applied Mathematics
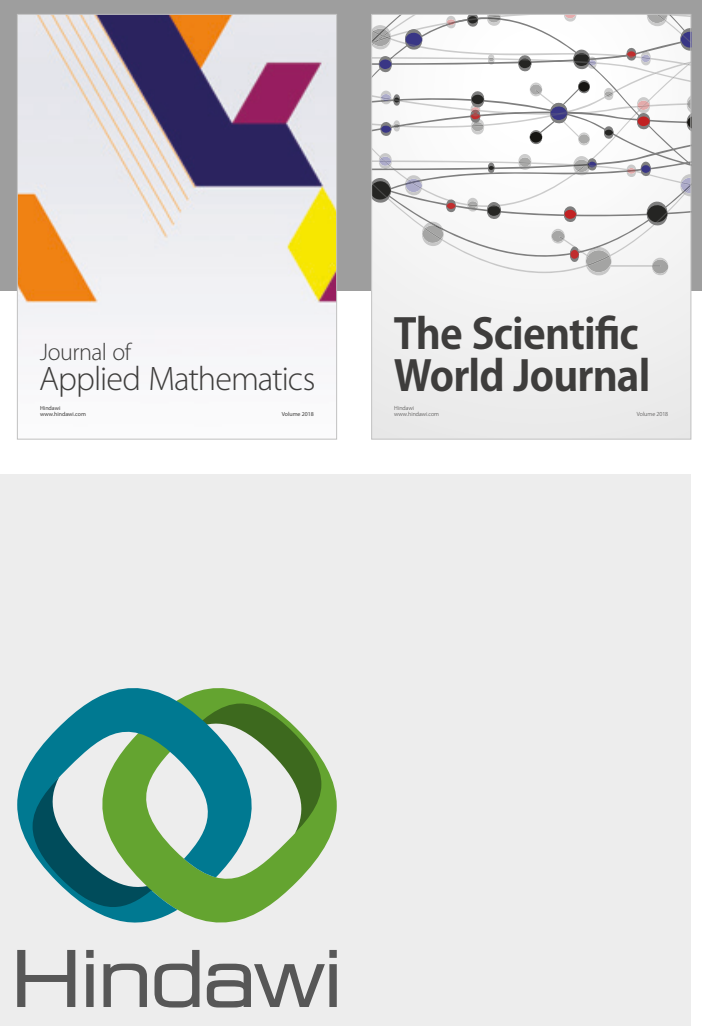

Submit your manuscripts at

www.hindawi.com

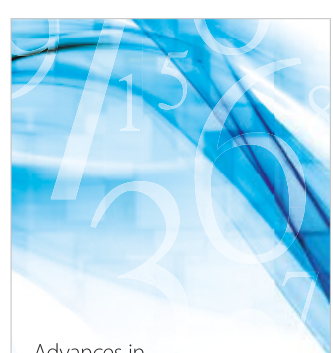

Advances in
Numerical Analysis
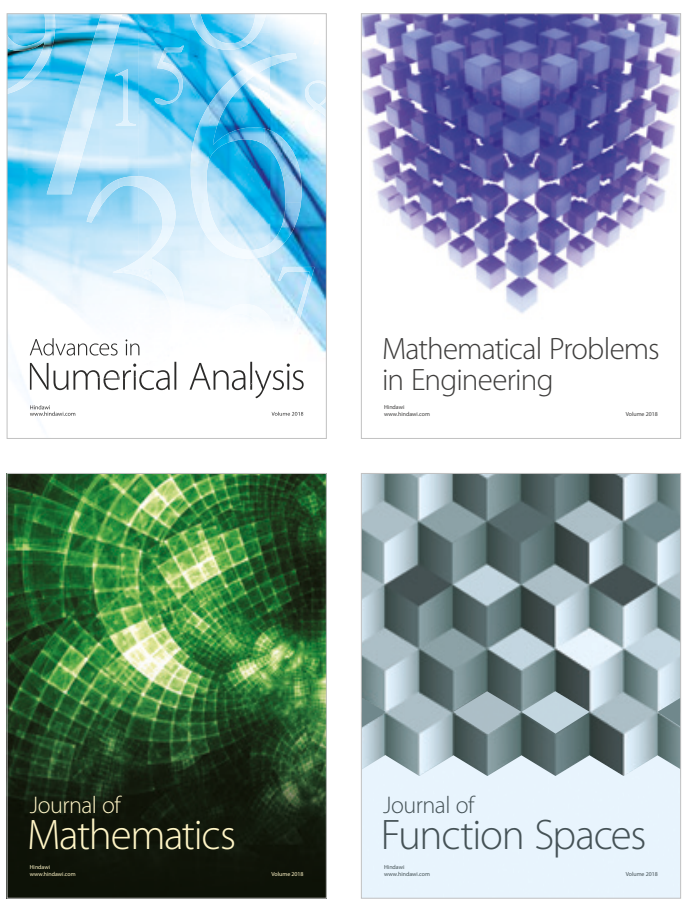

Mathematical Problems in Engineering

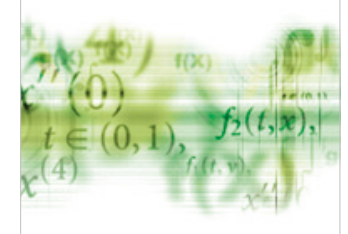

International Journal of

Differential Equations

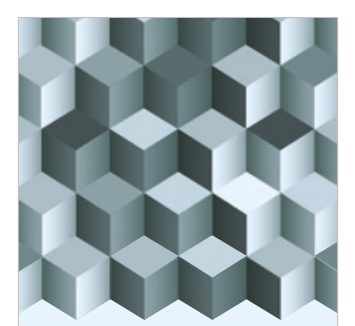

Journal of

Function Spaces

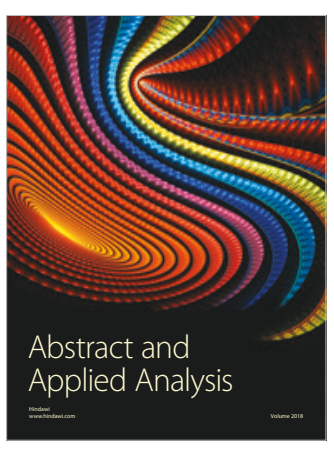

The Scientific

World Journal

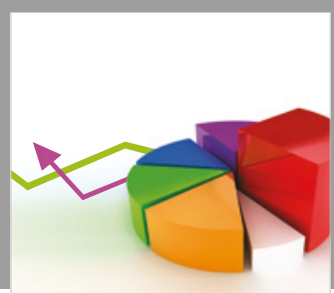

Journal of

Probability and Statistics
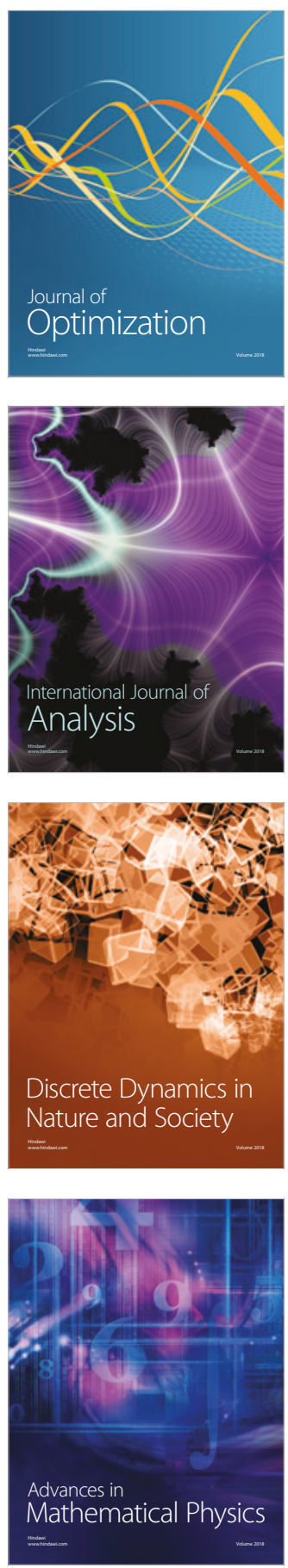